\title{
UBV PHOTOMETRY OF THE BRIGHT NUCLEI OF THE PLANETARY NEBULAE
}

\author{
E. B. Kostuakova, M.V. Savel'eva, O. D. Dokuchaeva, R. I. Noskova \\ (Sternberg State Astronomical Institute, Moscow, U.S.S.R.)
}

The data, which we have at present for the planetary nebulae central stars, are in general insufficient and uncertain. Even the photographic magnitudes of the brightest nuclei are usually given in the known catalogues with accuracies of $2^{\mathrm{m}}$ or $3^{\mathrm{m}}$. Therefore, the determination of central stars' magnitudes by the photoelectric method seems to be of great importance.

In the spring of 1966 we began photoelectric observations of the bright planetary nuclei at the Crimean Station of the Sternberg State Astronomical Institute. We used the $48-\mathrm{cm}$ parabolic reflector at the Cassegrain focus with a photometer employing an EMI-photomultiplier. The filters used gave an instrumental photometric system close to that of the UBV system.

For the first series of observations we selected nebulae of large angular diameters and relatively low surface brightness, but with central stars brighter than 13 . 5 . The radiation of the nebula itself was taken into account by measuring the nebular background in several points in the vicinity of the nucleus.

Correction for the atmospheric extinction was carried out by means of Nikonov's method. For this purpose the polar star HD 80354 (A0; V=9.76 $\pm 0.02, \mathrm{~B}-\mathrm{V}=$ $+0 \cdot 22 \pm 0 \cdot 01, \mathrm{U}-\mathrm{B}=+0 \cdot 11 \pm 0 \cdot 02$, according to our determinations) was selected as an atmospheric reference and observed several times every night.

Twelve members of the galactic cluster NGC 6633 were used as standards to reduce the instrumental photometric system to the UBV one. The colors B-V and U-B and the magnitudes $\mathrm{V}$ were taken from the work of Hiltner et al. (1958). The whole observation program was carried out from May until December of 1966.

Table 1 gives the results for the nuclei of 24 planetary nebulae: the colors B-V and U-B and the magnitudes $V$, with their root-mean-square deviations; the last column of the table shows the number of observations for each object.

The central star magnitudes of three nebulae - NGC 1501, 2371-2 and 6804 - are close to the limiting magnitude of the instrument used. Therefore, the results for these objects are of lower accuracy than for other nuclei.

Four nebulae - NGC 6543, 6572, 6853 and IC 3568 - have a relatively high surface brightness in comparison with the other nebulae of our list. In these cases the correction for the nebula radiation could not be done quite correctly. 
Table 1

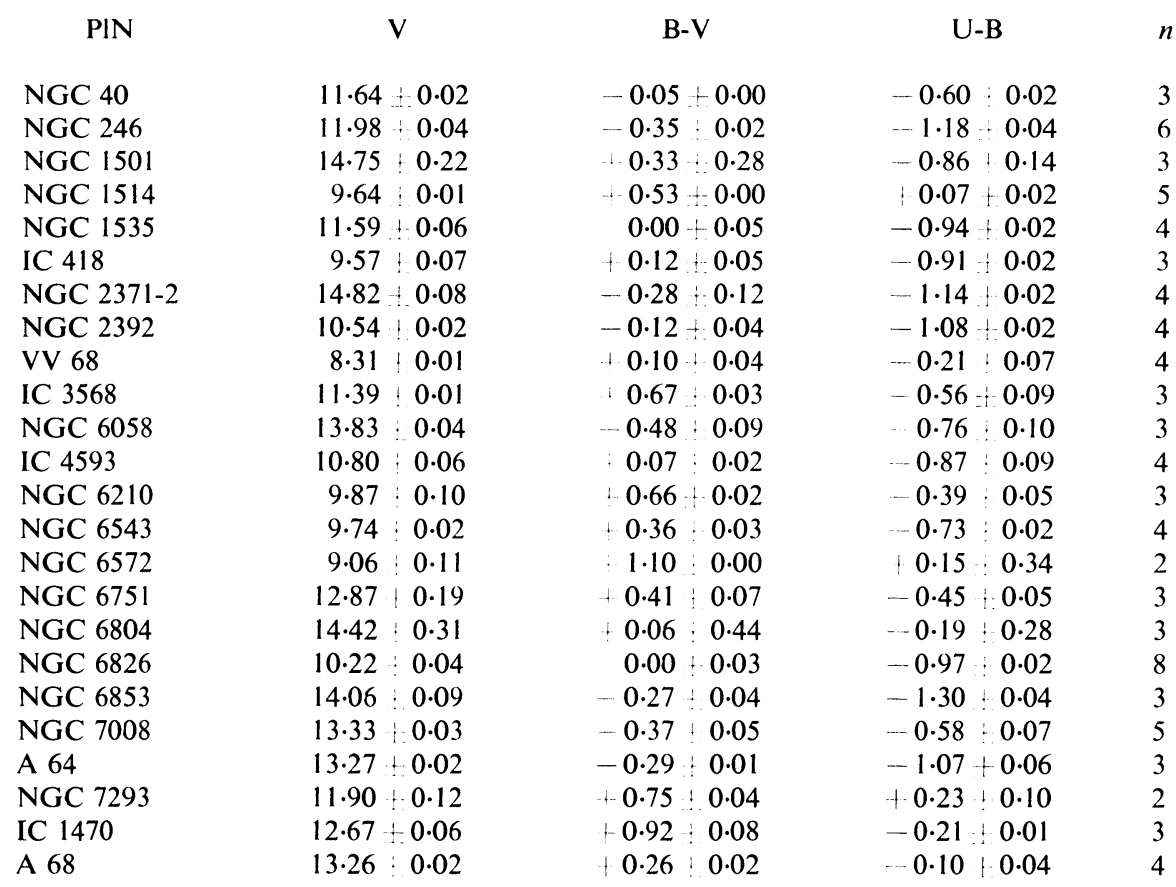

In the case of NGC 6210 , due to its small dimensions and high surface brightness, the correction for the nebular radiation was not done. The data concern the entire object and not its nucleus alone.

For the nebula NGC 7293 the identification of the central star seems to be doubtful. The results obtained can be demonstrated in Figure 1, where the color-color diagram is drawn for the central stars studied. The nuclei with photoelectric colors, determined by Abell (1966), are plotted in the figure as crosses for comparison. The figure also shows the curve of the main-sequence stars, the locus of unreddened black bodies and the reddening lines. We can see in this figure that the majority of nuclei are situated in the region of the hottest stars.

We can make an approximate correction for the interstellar extinction for many planetaries. After such a procedure many of their nuclei are also located in the region of the hottest stars. However, we can see that some planetaries remain in the region of yellow stars, even after an interstellar extinction correction. These mainly concern the above-mentioned doubtful planetaries and nuclei with possible duplicity. The most interesting objects in that aspect are: V-V 68, A-68, NGC 7293 and NGC 1514. We can point out that A-10, studied by Abell, also showed a yellow color. Kohoutek's (1968) recent work dealt with NGC 1514 in detail. 


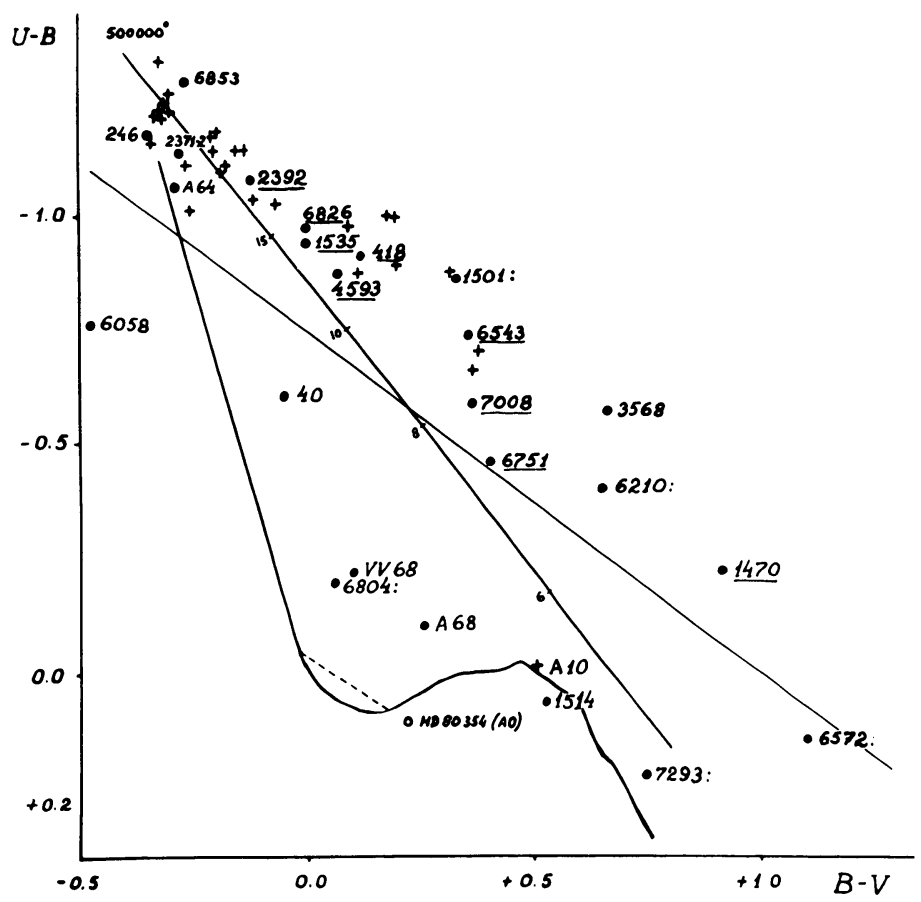

FIG. 1. Color-color diagram for central stars of planetary nebulae. Observations by Abell are shown by crosses, results of this investigation by filled circles. The fine lines show the locus of blackbody colors and the reddening slope. The underlined numbers show the nuclei which can reach the region of the hottest stars after an interstellar extinction correction.

Evidently, each of the above-mentioned planetaries needs additional and more detailed study; at any rate, this problem may be considered open to question. We intend to carry on with this work; the second series of observations have already begun.

\section{References}

Abell, G. O. (1966) Astrophys. J., 144, 259.

Hiltner, W.A., Iriarte, B., Johnson, H. L. (1958) Astrophys. J., 127, 539.

Kohoutek, L. (1968) in the present volume, p. 324.

\section{DISCUSSION}

W. Liller: I would like to ask Mrs. Kostjakova if she used special filters to suppress nebular radiations.

Kostjakova: We excluded the luminosity of the nebula in this way: We observed the background of the nebula with the same filters in several points, in the close vicinity of the nucleus. So we could exclude the luminosity of the nebula itself. In the majority of cases the background of the nebula was of the same order as the luminosity of the night sky. Only in a few cases (about three) was the luminosity of the nebulae larger. 